\title{
Teste de Habilidades Preditoras da Leitura: Normas de Habilidade para Crianças
}

\author{
Carla Alexandra da Silva Moita ${ }^{1,2}$, Émille Burity Dias ${ }^{2,3}$ \\ Universidade Federal da Paraíba, João Pessoa-PB, Brasil
}

RESUMO

O objetivo da investigação consistiu em construir os dados normativos das habilidades em consciência fonológica e memória visual do Teste de Habilidades Preditoras da Leitura (THPL) para crianças entre 4 e 8 anos. O instrumento avalia o nível de habilidade em consciência fonológica e memória visual, é de fácil aplicação, autoaplicável, adaptativo e informatizado. Participaram da investigação 404 crianças, meninas e meninos frequentando escolas da educação infantil ou fundamental. Foram conduzidas ANOVAs e análises post-hoc em função da escolaridade e idade, que as análises evidenciaram diferenças significativas. Os resultados revelaram que as crianças mais velhas e de séries mais avançadas possuem melhor habilidade no THPL do que as crianças mais novas e em séries iniciais. Foram utilizadas escalas de valores percentis, como medida normativa das habilidades avaliadas por ano escolar e idade. O instrumento poderá ser utilizado em contexto de pesquisa para análise do desempenho infantil em tarefas de elementos essenciais ao processo exitoso da leitura.

Palavras-chave: consciência fonológica; memória; psicometria; normas.

\section{ABSTRACT - Reading Predictive Skills Test: Skills Standards for Children}

The research objective was to construct normative data of the phonological awareness and visual memory skills for the Predictive Assessment of Reading (PAR) for children between 4 and 8 years of age. The instrument assesses skill level in phonological awareness and visual memory, is easy to apply, self-applied, adaptive and computerized. A total of 404 children attending primary or secondary school participated in the research. ANOVA and post-hoc analyses were conducted based on schooling and age, which showed significant differences. The results revealed that older children and those in higher grade levels are better skilled in PAR than younger children and those in earlier grades. Percentile value scales were used as normative abilities measure evaluated by school year and age. The instrument can be used in a research context to analyze children's performance in tasks of essential elements for successful reading.

Keywords: phonological awareness; memory; psychometry; norms.

\section{RESUMEN - Test de Habilidades Predictoras de Lectura: Normas de Habilidades para Niños}

El objetivo de esta investigación consistió en construir datos normativos sobre habilidades en la conciencia fonológica y la memoria visual del Test de Habilidades Predictoras de Lectura (THPL) para niños entre 4 y 8 años. Este instrumento es de fácil aplicación, autoaplicable, adaptado y computarizado, y evalúa el nivel de habilidades en la conciencia fonológica y en la memoria visual. Participaron 404 niños de ambos sexos que frecuentaban jardín de infantes y enseñanza primaria. Se llevaron a cabo ANOVA análisis de varianza y post-hoc en función de escolaridad y edad; los resultados de tales análisis evidenciaron diferencias significativas. Los niños de más edad y de series más avanzadas poseen mejor habilidad en el THPL, que los de menor edad y de series iniciales. Fueron utilizadas escalas de valores porcentuales como medida normativa de las habilidades evaluadas por año escolar y edad. Este instrumento puede utilizarse en el contexto de investigación para análisis del desempeño infantil en tareas de elementos esenciales al proceso exitoso de la lectura. Palabras clave: conciencia fonológica; memoria; psicometría; normas.

Será que existe alguma dúvida acerca da importância da leitura para o desenvolvimento, o crescimento e a capacidade de relacionamento do ser humano? Acredita-se que a premissa "ler é fundamental" é verdadeira e indiscutível. A leitura é uma criação humana: o homem criou a leitura e, ao longo dos séculos, vem ensinando de geração a geração como se lê.
A leitura é aprendida e, para tanto, deve ser ensinada. Para que a leitura ocorra de forma satisfatória e cumpra com a missão de abrir as "portas" para outras aprendizagens, um complexo e imbricado sistema é ativado; esse sistema envolve desde quem lê, o que lê, para que lê, aonde lê e de onde lê.

${ }^{1}$ Os autores agradecem ao CNPQ pelo apoio e financiamento ao projeto.

2 Endereço para correspondência: Rua Giácomo Porto, 145, apto 1601, 58032-110, João Pessoa-PB. Tel.: (83) 99982-7911. E-mail: carlamoitaminervino@gmail.com 3 Os dados apresentados fazem parte parcialmente da dissertação da Émille Burity Dias apresentada ao Programa de Pós Graduação em Neurociência e Comportamento. 
A complexidade desse sistema e os múltiplos fatores envolvidos justifica o interesse de muitos pesquisadores, de diversas áreas do conhecimento, que visam compreender a leitura: desde a ação de ler à ação de ensinar a ler. As pesquisas em âmbito nacional e internacional (e.g., Liberman, 1971, 1974, 1979; Moraes, 1979; Ehri, 1978, 1979, 1998; Capovilla, 1995; Capellini, 1999; Minervino, 2000; Viana, 2002; Cruz, 2007), desde a década de 70 até os tempos atuais, vem revelando que o ato de ler não é aprendido apenas no momento da alfabetização; a literacia tem início muito antes do momento escolar do primeiro ano. O sistema que permite o ato de ler é ativado muito precocemente.

Nesse sentido, surgem as pesquisas com enfoque nas habilidades cognitivas preditoras da leitura, visto que estas apresentam um suporte para que a leitura inicie e se processe de maneira eficaz. Conforme Capellini et al. (2009) estudos internacionais referem-se à necessidade da presença de cinco elementos, em crianças pré-escolares, para que a aprendizagem da leitura ocorra com eficiência, são eles: consciência fonológica, conhecimento dos fonemas, fluência verbal, vocabulário e compreensão. Além da influência da memória fonológica e visual em todo o processo de aquisição da leitura. Estudos como os de Gerber e Tomitch (2008), Barbosa, Bernardes, Misorelli, e Chiappetta (2010) e Fuchs et al. (2012) verificaram que a memória de trabalho (fonológica), permite que o leitor recorde-se das regras de conversão grafo-fonêmicas durante o processo de decodificação das palavras.

A memória de trabalho fonológica realiza o estoque temporário e processamento concomitante de informações e mensagens, nesse sentido, estudos associam essa habilidade à competência/compreensão leitora, pois, o armazenamento temporário de segmentos do texto ou apenas palavras é fundamental para a seleção, interpretação e evocação das informações, logo, da compreensão leitora (Nevo \& Bar-Kochva, 2015; Demoulin \& Kolinsky, 2015; Fonsaca, 2013). Já a memória visual está relacionada com o reconhecimento dos traços que constituem as letras do sistema alfabético, conforme Dahaene e Cohen (2011), a criança deve inicialmente ser capaz de diferenciar a direção dos traços para esquerda ou direta, para cima ou para baixo. Na fase pré-leitora, Dahaene (2012) afirma que a criança utiliza as pistas visuais para atribuir significado as letras e posteriormente as palavras. Essa capacidade é fomentada pela atuação da memória visual.

A importância do conceito de consciência fonológica na teorização sobre aquisição da leitura não pode ser subestimada e, desde a pesquisa de Liberman, em 1971, essa habilidade tem sido vista como chave para desvendar o complexo processo pelo qual as crianças aprendem a relação entre escrita e leitura das palavras (Castles \& Coltheart, 2004). A consciência fonológica é considerada como a habilidade de reconhecer e manipular os sons da língua (Worrell, Watkins, Runge, \& Hall, 2002).
Embora haja consenso entre os pesquisadores que a consciência fonológica é significativamente relacionada com as fases iniciais de leitura e escrita, Schneider, Küspert, Roth, Visé, e Marx (1997) pontuam que a relação causal entre a consciência fonológica no início e posterior leitura eficiente e habilidades de ortografia tem sido controversa; uma possível interpretação da evidência de correlação é que a consciência fonológica precede a aquisição da leitura. De forma alternada, aprender a ler pode ser um pré-requisito para tornar-se consciente da estrutura fonológica das palavras. A terceira interpretação é que a relação é recíproca: a consciência fonológica no início facilita a visão sobre o código alfabético, que por sua vez melhora o nível de consciência fonológica. Segundo os autores, um dos estudos, com pré-escolares, mais sofisticado e completo foi realizado por Lundberg, Frost e Petersem, em 1988. Esses pesquisadores realizaram um estudo longitudinal com 400 crianças, utilizando um programa de treinamento que consiste em jogos e exercícios metalinguísticos. O treinamento foi realizado durante o último ano da pré-escola e durou cerca de 8 meses. Os autores concluíram que a consciência fonológica pode ser desenvolvida antes e independentemente da habilidade da leitura; e a consciência fonológica facilita a posterior aquisição da alfabetização.

Para Torgesen (2002) e Nelson (2008), não existem dúvidas que um dos achados mais importantes, nos últimos 30 anos, é o impacto da consciência fonológica para o desenvolvimento eficaz da leitura. Os autores consideram a consciência fonológica como o melhor preditor das posteriores habilidades da leitura. Entretanto, Foorman, Herrera, Petscher, Mitchell, e Truckenmiller (2015) revelam que treinamentos em consciência fonológica realizados entre a pré-escola e o $1^{\circ}$ ano predizem bom rendimento em leitura no $3^{\circ}$ ano e nos demais anos escolares, porém treinamentos realizados a partir do $2^{\circ}$ ano já não possuem caráter preditor tão significativo.

Diante da importância do tema e da existência de poucos instrumentos adaptativos computadorizados brasileiros para a avaliação de habilidades, que são consideradas pela literatura como preditoras da aquisição leitura, foi elaborado o teste de habilidades preditoras da leitura (THPL). Para Carson, Gillon, e Boustead (2011), os instrumentos adaptativos computadorizados são eficazes e mais rápidos, além disso, estudos como os de Bridges e Catts (2011), Partanen e Siegel, (2014) e os de Gellert e Elbro (2015) referendam que boas baterias para análise de consciência fonológica reduzem substancialmente a incidência de identificação de falsos positivos para o risco de dificuldades de leitura.

O Teste de Habilidades Preditoras da Leitura - THPL (Minervino, Moita, Assis, Lucena, Dias, \& Fonsaca, 2013) é voltado pra crianças a partir da pré-escola. Sua criação surgiu, portanto, da necessidade de testes informatizados, adaptativos, autoaplicáveis, com aplicação, correção e interpretação de resultados 
práticos, de fácil manejo por parte dos examinados. Trata-se de um teste informatizado desenvolvido com base na teoria dos testes adaptativos informatizados e na teoria de resposta ao item.

O instrumento disponibiliza questões autoaplicáveis a serem respondidas pela criança individualmente com o auxílio de um dispositivo móvel e posterior divulgação do desempenho individual da criança. Os resultados ficam disponíveis para o usuário do sistema (aplicador). O THPL possui, até o momento, quatro tarefas: rima, aliteração, segmentação (que avaliam a consciência fonológica) e memória visual, desenvolvidas para pré-escolares. $\mathrm{O}$ aplicador acessa o sistema no ambiente virtual, cadastra-se e, após análise do administrador, recebe login e senha, uma vez realizado esse procedimento, ele poderá utilizar o instrumento. Até o momento, o instrumento só está disponível para pesquisadores.

Ao iniciar, o aplicador deverá preencher os dados de identificação da criança e, em seguida, o dispositivo móvel deverá ser passado para as mãos da criança, que executa o teste sozinha. Os itens são apresentados à criança um a um e ela deverá apenas escutar e tocar na resposta que acredita ser a correta.

Todas as crianças iniciam o THPL com itens da mesma dificuldade (destaca-se que, para o desenvolvimento do instrumento, foi utilizado um banco de itens com vários níveis de dificuldade), dessa forma, o instrumento considera que, no início da execução da tarefa, todas as crianças partem do mesmo nível de dificuldade, porém, na medida em que a criança responde aos itens, estes irão variar de nível de dificuldade. O instrumento seleciona o item conforme a resposta dada pela criança, se a criança acerta ao primeiro item um novo será apresentado com um nível de dificuldade maior, entretanto, se a criança responder de forma incorreta acontecerá o inverso: surgirá um item de menor dificuldade, nesse sentido, a habilidade da criança irá construir o instrumento (Moita, 2013; Dias, 2013; Pereira, 2013; Fonsaca, 2013).

A resposta dada ao item é dicotômica (acerto/erro). Em virtude da adaptação do instrumento à habilidade da criança, cada uma responderá a um grupo de itens diferente. Caso duas crianças estejam realizando as tarefas ao mesmo tempo, é muito provável que cada uma delas irá responder a um grupo de itens diferente.

Por fim, fica disponível ao aplicador: a identificação do examinando (nome, sexo, idade, escola, ano escolar) e informações adicionais (se alguém lê para ela, se possui livros em casa). Essas informações são obtidas no início da tarefa a partir do preenchimento prévio do aplicador (Figura 1). Além disso, também ficará disponível a data de aplicação, tempo de execução para cada tarefa e para cada item que fora respondido pela criança, a habilidade em cada tarefa, a quantidade de itens respondidos, o índice de dificuldade, discriminação e acerto casual de cada item respondido e a porcentagem de acerto (essas informações são obtidas após a execução das tarefas). $\mathrm{O}$ desempenho individual é apresentado em valores/escores e em gráficos.

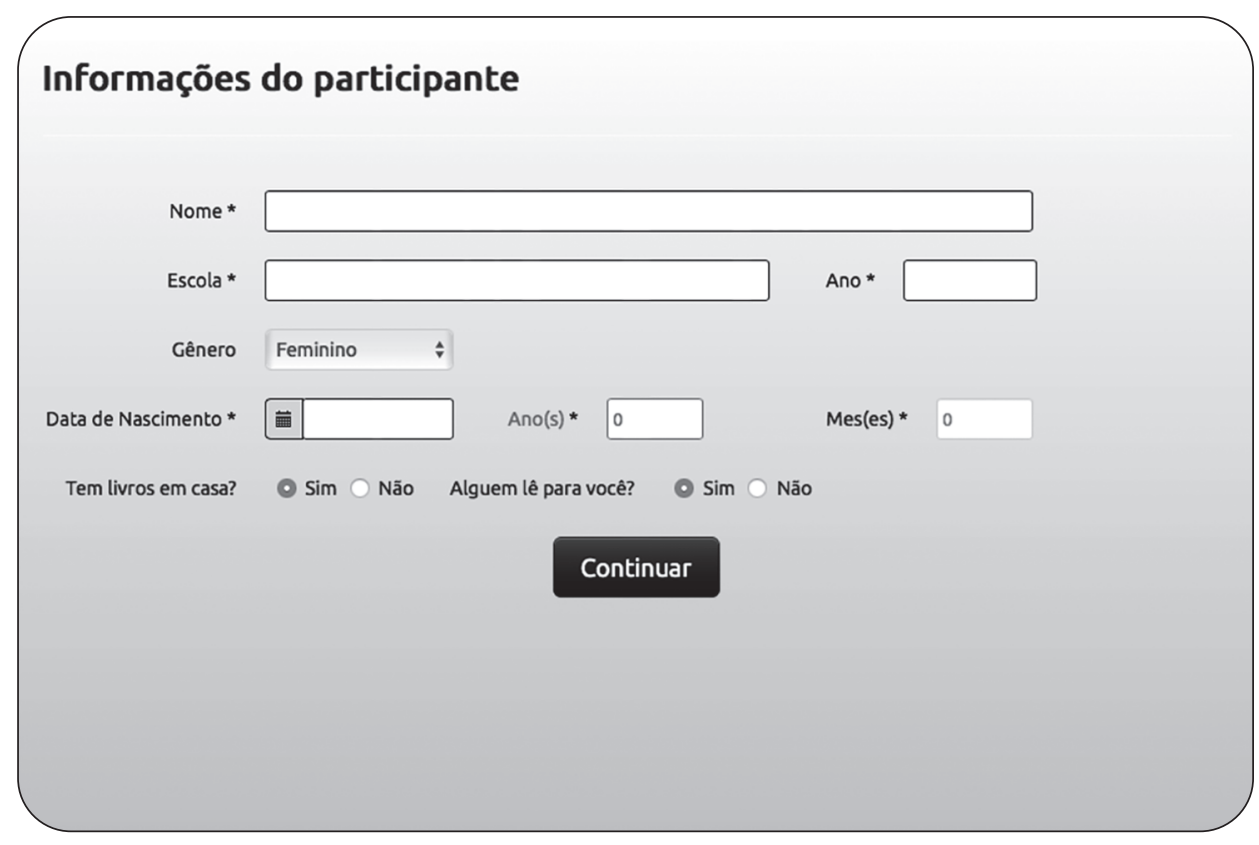

Figura 1. Tela de identificação da criança que responderá ao THPL (preenchimento obrigatório a ser realizado pelo aplicador) 
Conforme Moita (2013), o THPL apresenta valores de consistência interna satisfatórios, apresenta coeficiente alfa de Cronbach igual a 0,87 para substestes de consciência fonológica (rima, aliteração, segmentação) e 0,93 para subteste de memória. Evidências de validação de construto do THPL foram apresentados por Moita, Minervino \& Chambel (2014), estes demonstraram que os resultados foram significativos nas análises de esferecidade de Bartlett $(p<0,05)$ e índice de KMO (Kaiser-Meyer-Olkin) igual a 0,91, o que indicou que os dados poderiam ser submetidos à análise fatorial. O critério de Kaiser-Guttman indicou a presença de dois fatores com autovalor $>1$. A informação foi confirmada pelo a aplicação do método das análises paralelas. Os autores utilizaram o método de rotação de fatores oblíqua Promax e consideraram como critério mínimo o valor de 0,4 . Foram reveladas evidências de validade de construto mediante análises realizadas com a análise fatorial exploratória, destaca-se que foi realizada comparação com a literatura, ou seja, estrutura teórica sobre consciência fonológica e memória visual; os resultados confirmaram a dimensionalidade do THPL em dois fatores.

Ainda, nos estudos de Moita et al. (2014), foram apresentadas as descrições dos itens do THPL conforme o modelo de três parâmetros: a dificuldade, a discriminação e a probabilidade de resposta dada ao acaso. Os programas estatísticos utilizados foram: o programa BILOG-MG, que permite a análise de mais um grupo de respondentes, e o programa XCALIBRE para análise do ajuste dos modelos de três parâmetros pela estimação marginal de máxima verossimilhança, com a finalidade de verificar a precisão do instrumento, a correlação item-theta e os ajustes dos itens em relação à escala. Conforme os resultados apresentados pelos autores, observa-se que os itens do THPL (consciência fonológica e memória de trabalho) foram capazes de determinar as diferentes habilidades no desempenho das crianças; assim como verificar o nível de dificuldade do item (percebe-se a presença itens fáceis, medianos e difíceis), além de ser capaz de identificar a probabilidade de a resposta ser dada ao acaso em cada item. Todos os valores analisados dos três parâmetros foram considerados satisfatórios.

Em resumo, o THPL foi considerado um instrumento válido e preciso, no entanto, ainda não possui valores normativos de referência. Este estudo teve por objetivo estabelecer a normatização do Teste de Habilidades Preditoras da Leitura. A normatização de um teste refere-se ao estabelecimento de padrões de interpretação de um escore de determinado indivíduo, além de fazer parte da padronização do teste (Pasquali, 2001). No processo de normatização, os escores brutos são transformados em médias a fim de indicar a posição do indivíduo na amostra normativa, bem como avaliar o desempenho em relação aos demais (Anastasi \& Urbina, 2000). Especificamente, objetivou-se identificar diferenças entre os grupos: pré-escola, $1^{\circ}, 2^{\circ}$ e $3^{\circ}$ ano para habilidades de rima, aliteração, segmentação e memória visual.

\section{Método}

\section{Participantes}

Para a obtenção de dados normativos do THPL, foram considerados os dados do censo populacional brasileiro mais recente, de 2010 (IBGE, 2010). A população-base para o processo de seleção da amostra foi de crianças recenseadas de 4 a 8 anos de idade, que frequentavam a educação infantil.

Foram consideradas as seguintes informações para a constituição da amostra (1) densidade demográfica; (2) crianças recenseadas moradoras de zona urbana e (3) grau de desenvolvimento socioeconômico das cidades brasileiras, conforme os dados do Índice de Desenvolvimento Humano - IDH. Foi adotado o IDH (2010) composto por três fatores: renda, longevidade e educação. Ao considerar tais critérios, foi verificado a existência de 7.063.551 crianças na faixa etária entre 4 e 8 anos, frequentando escolas e morando na zona urbana das cidades brasileiras. Em seguida, foram selecionados os municípios em cada região que, além do IDHM, foram também utilizados os seguintes critérios: (a) as cidades selecionadas deverão contemplar os maiores estados da região; (b) estados com maiores e menores IDH do país; (c) disponibilidade de aplicadores no município e (d) a facilidade de acesso à internet, para atender a especificidade de aplicação do instrumento.

A partir desses critérios (contemplar os maiores estados da região e o IDH maior e menor do pais), foi observado que os estados com maiores IDH $(0,749$ a $0,824)$ do país encontram-se no sudeste brasileiro e com os menores índices $(0$ a 0,660$)$ no Nordeste. Em relação à disponibilidade de aplicadores no município e facilidade de acesso à internet, foram considerados municípios dos estados de São Paulo e da Paraíba. Em São Paulo, foram verificadas em torno de 1,8 milhão de crianças matriculadas e, na Paraíba, 121.590 crianças (IBGE, 2010). Em seguida foram consideradas apenas as crianças do município de São Paulo e João Pessoa. Convém ressaltar que a primeira etapa da normatização do THPL considerou apenas as crianças do município de João Pessoa (total de 19.806 crianças). Os dados referentes as crianças de São Paulo ainda estão sendo analisadas.

O cálculo amostral considerou o tamanho da população (19.806 crianças), o erro amostral de 5\%, o nível de confiança de $95 \%$ e a distribuição da população heterogênea, assim, o tamanho da amostra previsto foi de 377 crianças. A técnica de amostragem probabilística foi utilizada, considerando as escolas do município de João Pessoa com acesso à internet.

Participaram da investigação 404 crianças com idades entre 4 e 8 anos (média de idade $=6,02 ; D P=1,28$ ), 
sendo 218 (54\%) meninas e 186 (46\%) meninos. Os participantes eram de escolas públicas $(43,8 \%)$ e privadas $(56,2 \%)$, estudantes da educação infantil $(28,2 \%), 1^{\circ}$ ano $(37,4 \%), 2^{\circ}$ ano $(16,8 \%)$ e $3^{\circ}$ ano $(17,6 \%)$. Os critérios de inclusão para a participação neste estudo foram: (a) Assinatura dos Termos de Anuência (diretores de escola), Consentimento (pais e/ou responsáveis) e Assentimento (menor); (b) ausência de comprometimentos físicos e/ ou emocionais que pudessem impedir a resposta no dispositivo móvel; (c) responder a todas as tarefas propostas no THPL.

\section{Instrumento}

O THPL (Minervino et al., 2013) é constituído por três tarefas de consciência fonológica no nível de sílaba: (a) segmentação silábica; (b) identificação de sílaba inicial e (c) identificação de rima; e uma tarefa de memória visual. A apresentação de cada item é realizada oralmente e a criança deve escolher a resposta que achar pertinente. Exemplo: na tarefa de rima é apresentada a figura do PATO e em seguida verbalizada a palavra PATO; terminada a verbalização são apresentadas três figuras em que apenas uma rima com PATO; a criança deverá escolher a figura que termina com o mesmo som de PATO.

No formato informatizado (Moita, 2013), possui 307 itens distribuídos nas três tarefas. É autoaplicável, as orientações e as figuras são verbalizadas no próprio sistema, não sendo necessária a orientação do aplicador. Apenas os dados de identificação devem ser preenchidos pelo aplicador.

Cada criança responde a uma quantidade individualizada de itens que depende do acerto/erro no decorrer da execução tarefa. No final da execução, estarão disponíveis os dados da identificação e o desempenho individual da criança. Em relação à análise do desempenho individual, são apresentados o valor calculado para a habilidade da criança em cada tarefa; a média dos valores da habilidade de acordo com a resposta em cada item, o percentual de acerto por tarefa considerando o número total de itens respondido pela criança; o escore bruto do número de acertos por número de itens respondidos; valor médio da habilidade no fator (1) consciência fonológica e no fator (2) memória visual, além do tempo de execução, do total de tarefas, de cada tarefa e de cada item.

Ainda em relação ao desempenho, são apresentados quatro gráficos referentes a cada tarefa onde é possível verificar a evolução da dificuldade do item versus a habilidade da criança calculada durante a execução das tarefas. No gráfico, é possível acessar os parâmetros que descrevem cada item (a dificuldade, a discriminação e a probabilidade de acerto ao acaso) respondido pela criança.

Foram utilizados dispositivos móveis para a aplicação do THPL: modelo com WiFi e 3G, armazenamento de $16 \mathrm{~GB}$, ecrã panorâmico brilhante multi-touch de 9,7 polegadas (diagonal) com retroiluminação LED e tecnologia IPS, resolução de 1024 por 768 a 132 píxeis por polegada (ppp), áudio resposta de frequência de 20 $\mathrm{Hz}$ a $20.000 \mathrm{~Hz}$.

\section{Procedimentos}

Os dados foram coletados em escolas públicas e particulares situadas no perímetro urbano da cidade de João Pessoa, Paraíba. A coleta foi realizada por pesquisadores experientes na utilização do instrumento. O THPL foi aplicado em ambiente a parte da sala de aula, de forma individual. O preenchimento dos dados de identificação da criança foi realizado pelo pesquisador e, em seguida, o dispositivo móvel era passado para as mãos da criança, que tinha a sua disposição um fone intra-auricular. $\mathrm{O}$ tempo médio de execução foi de 12 minutos.

$\mathrm{O}$ presente estudo respeitou e considerou todos os padrões éticos de conduta. A identificação por parte das crianças foi substituída por números de protocolo, de maneira que não houvesse identificação e/ou constrangimento para os sujeitos amostrais envolvidos na pesquisa, conforme a Resolução n ${ }^{\circ}$ 466/12 do Conselho Nacional de Saúde/MS e suas complementares, outorgada em 13 de junho de 2013, visando assegurar os direitos e deveres que dizem respeito à comunidade científica, ao(s) sujeito(s) da pesquisa e ao Estado, projeto aprovado pelo Comitê de Ética do Hospital Universitário Lauro Wanderley (parecer no. 346.404).

As análises foram efetuadas com o auxílio do pacote estatístico IBM SPSS Statistics (versão 21.0 para Windows). A análise da habilidade das crianças na execução do THPL foi realizada separadamente para cada tarefa que compõe o instrumento. Foi efetuada análise estatística descritiva, aferição de frequência absoluta, médias, medianas, desvios padrão. Para as análises, foram considerados os valores de habilidade na tarefa. Com auxílio do teste de KomolvorogSmirnov, foi possível constatar que os dados se distribuem normalmente $(p>0,05)$, por ano escolar e por grupos etários. A normatização foi realizada conforme análise dos percentis. Para verificar diferenças entre grupos, foi utilizada a Análise de Variância (ANOVA).

\section{Resultados}

As informações sobre os participantes e as análises descritivas das habilidades, nas tarefas do THPL, são apresentadas na Tabela 1. Foram conduzidas ANOVAs e análises post-hoc (Teste de Tukey) das medidas do THPL em função da escolaridade e idade, tais análises evidenciaram diferenças significativas entre as habilidades nas tarefas das crianças investigadas (Tabela 2).

Para verificar a homoscedasticidade, utilizou-se o teste de Levene. Foi verificado que as variâncias da distribuição das habilidades por ano escolar são semelhantes $[F(3,400)=0,338 ; p=0,798]$. A habilidade na tarefa de aliteração diferiu significativamente entre os anos escolares $[F(3,400)=19,073 ; p<0,001]$, O teste de Tukey 
(DHS) indicou que as habilidades das crianças da pré-escola foram significativamente inferiores àquelas apresentadas pelas crianças dos $1^{\circ}, 2^{\circ}$ e $3^{\circ}$ anos. As habilidades diferiram entre o $1^{\circ}$ ano e o $2^{\circ}$ ano $(p=0,007)$. No entanto, não houve diferença significativa entre as habilidades das crianças do $2^{\circ}$ e $3^{\circ}$ ano $(p=0,953)$.

Tabela 1

Média da Habilidade na Tarefa, Desvio Padrão das Habilidades das Crianças por Ano Escolar e Idade

\begin{tabular}{|c|c|c|c|c|c|c|c|c|c|c|c|}
\hline \multirow{3}{*}{$\begin{array}{c}\text { Ano } \\
\text { Escolar/Idade }\end{array}$} & \multirow{3}{*}{ N } & \multicolumn{8}{|c|}{ Tarefas } & \multirow{2}{*}{\multicolumn{2}{|c|}{$\begin{array}{c}\text { Consciência } \\
\text { Fonológica Total }\end{array}$}} \\
\hline & & \multicolumn{2}{|c|}{ Aliteração } & \multicolumn{2}{|c|}{ Segmentação } & \multicolumn{2}{|c|}{ Rima } & \multicolumn{2}{|c|}{ Memória } & & \\
\hline & & M & DP & M & DP & M & $D P$ & M & DP & M & DP \\
\hline \multicolumn{12}{|l|}{ Pré-Escola } \\
\hline 4 anos & 55 & 0,544 & 1,400 & $-0,548$ & 2,115 & $-0,388$ & 1,385 & 0,511 & 1,788 & $-0,141$ & 1,126 \\
\hline 5 anos & 59 & 0,434 & 1,273 & $-0,618$ & 2,117 & $-0,329$ & ,963 & 0,853 & 1,794 & $-0,159$ & 0,908 \\
\hline Total & 114 & 0,487 & 1,331 & $-0,584$ & 2,107 & $-0,357$ & 1,181 & 0,688 & 1,791 & $-0,150$ & 1,015 \\
\hline \multicolumn{12}{|l|}{$1^{\circ}$ ano } \\
\hline 5 anos & 39 & 0,736 & 1,635 & $-0,963$ & 1,936 & 0,310 & 1,400 & 1,240 & 1,498 & 0,027 & 1,217 \\
\hline 6 anos & 101 & 1,216 & 1,395 & $-0,092$ & 1,922 & 0,559 & 1,210 & 1,336 & 1,619 & 0,540 & 1,113 \\
\hline 7 anos & 11 & 0,606 & 1,056 & $-0,261$ & 1,953 & $-0,577$ & 1,145 & 0,578 & 1,708 & $-0,347$ & 1,322 \\
\hline Total & 151 & 1,048 & 1,452 & $-0,330$ & 1,952 & 0,412 & 1,284 & 1,256 & 1,596 & 0,343 & 1,185 \\
\hline \multicolumn{12}{|l|}{$2^{\circ}$ ano } \\
\hline 7 anos & 62 & 1,623 & 1,213 & 0,310 & 1,499 & 0,935 & 1,192 & 2,130 & 1,254 & 0,943 & 0,991 \\
\hline 8 anos & 6 & 2,168 & 1,046 & $-0,385$ & 2,152 & 0,997 & 0,660 & 2,555 & 0,831 & 0,886 & 0,824 \\
\hline Total & 68 & 1,671 & 1,203 & 0,249 & 1,559 & 0,941 & 1,152 & 2,168 & 1,224 & 0,938 & 0,972 \\
\hline \multicolumn{12}{|l|}{$3^{\circ}$ ano } \\
\hline 8 anos & 71 & 1,789 & 1,055 & 0,549 & 1,479 & 1,146 & 1,199 & 2,368 & 1,000 & 1,151 & 0,924 \\
\hline
\end{tabular}

Tabela 2

Contrastes Encontrados nas Habilidades das Crianças nas Tarefas do THPL entre os Anos Escolares (Teste Post-Hoc Tukey DHS)

\begin{tabular}{|c|c|c|c|}
\hline & Pré-escola & $1^{\circ}$ ano & $2^{\circ}$ ano \\
\hline \multicolumn{4}{|c|}{ Tarefa: Aliteração } \\
\hline \multicolumn{4}{|c|}{ Pré-escola } \\
\hline $1^{\circ}$ ano & $p=0,004$ & & \\
\hline $2^{\circ}$ ano & $p<0,000$ & $p=0,007$ & \\
\hline $3^{\circ}$ ano & $p<0,000$ & $p=0,001$ & $p=0,953$ \\
\hline \multicolumn{4}{|c|}{ Tarefa: Segmentação } \\
\hline \multicolumn{4}{|c|}{ Pré-escola } \\
\hline $1^{\circ}$ ano & $p=0,689$ & & \\
\hline $2^{\circ}$ ano & $p=0,019$ & $p=0,146$ & \\
\hline $3^{\circ}$ ano & $p<0,000$ & $p=0,006$ & $p=0,779$ \\
\hline \multicolumn{4}{|c|}{ Tarefa: Rima } \\
\hline \multicolumn{4}{|c|}{ Pré-escola } \\
\hline $1^{\circ}$ ano & $p<0,000$ & & \\
\hline $2^{\circ}$ ano & $p<0,000$ & $p=0,017$ & \\
\hline $3^{\circ}$ ano & $p<0,000$ & $p<0,000$ & $p=0,753$ \\
\hline \multicolumn{4}{|c|}{ Tarefa: Memória } \\
\hline \multicolumn{4}{|c|}{ Pré-escola } \\
\hline $1^{\circ}$ ano & $p=0,014$ & & \\
\hline $2^{\circ}$ ano & $p<0,000$ & $p<0,000$ & \\
\hline $3^{\circ}$ ano & $p<0,000$ & $p<0,000$ & $p=0,864$ \\
\hline
\end{tabular}

$\mathrm{Na}$ tarefa de segmentação, como função do ano escolar, a habilidade foi significativamente diferente $[F(3,400)=6,901, p<0,001]$. O teste de Tukey (DHS) indicou que as habilidades das crianças da pré-escola foram significativamente inferiores daquelas apresentadas pelas crianças dos 2o e 3o anos escolares, entretanto, não ocorreu diferença entre as habilidades na pré-escola e no 1o ano $(p=0,689)$. O mesmo aconteceu entre as crianças do $1 \mathrm{o}$ ano e do $2 \mathrm{o}$ ano $(p=0,146)$ e as habilidades das crianças do 2 o e 3 o ano $(p=0,779)$.

As habilidades na tarefa de rima foram significativamente diferentes entre todos os anos escolares $[F(3,400)=27,978, p<0,001]$, com exceção das habilidades entre os anos $2^{\circ}$ e $3^{\circ}(p=0,753)$. Na tarefa de memória, ocorreram diferenças significativas entre os anos escolares $[F(3,400)=24,268, p<0,001]$, porém também não ocorreram diferenças significativas entre os $2^{\circ}$ e $3^{\circ}$ anos $(p=0,864)$.

Esses resultados sugerem que as crianças do $3^{\circ}$ e $2^{\circ}$ anos apresentam uma melhor habilidade no THPL do que aquelas da pré-escola e $1^{\circ}$ ano. Nesse sentido, buscou-se ainda refinar as diferenças na habilidade nas tarefas do THPL com a finalidade de evidenciar se este é sensível para discriminar as habilidades das crianças no período pré-alfabetização e alfabetização daquelas crianças que estavam cursando o $2^{\circ}$ e $3^{\circ}$ Anos (pós-alfabetização), 
para tanto, as crianças foram novamente agrupadas e foi realizado o teste $t$ de Student. Foram verificadas diferenças significativas em todas as tarefas do THPL $(p<0001)$. As crianças de $2^{\circ}$ e $3^{\circ}$ anos demonstraram uma melhor pontuação no THPL do que aquelas que estavam na pré-escola e $1^{\circ}$ Ano.

A relação entre as habilidades nas tarefas do THPL, considerando a idade das crianças, também foi analisada. Foi realizada a ANOVA, e os resultados mostram a existência de diferenças significativas nas habilidades das tarefas de aliteração $[F(4,399)=13,903, p<0,001]$, segmentação $[F(4,399)=6,192, p<0,001]$, rima $[F(4,399)=17,460$, $p<0,001]$ e memória $[F(4,399)=16,109, p<0,001]$. Na tarefa de aliteração, o teste de Tukey (DHS) indicou que as habilidades das crianças de 4 anos foram significativamente inferiores que aquelas apresentadas por crianças de 6,7 e 8 anos $(p=0,02, p=0,001, p<0,001)$, não ocorreu diferença entre as habilidades aos 4 e 5 anos $(p=1,00)$. Padrão semelhante aconteceu na tarefa de rima e memória. No entanto, na tarefa de segmentação, revela-se que as crianças começaram a apresentar melhores resultados aos 7 anos. Não foi verificada diferença significativa entre os 4,5 e 6 anos.

Foram analisadas habilidades nas tarefas considerando o tipo de escola (públicas e privadas), para tanto foi conduzido um teste $t$ para amostras independentes, e os resultados indicam existir algumas diferenças significativas, considerando a habilidade em cada tarefa por ano escolar. Em relação ao grupo de crianças da pré-escola (escolas públicas versus privadas) houve diferenças significativas nas tarefas de aliteração $[t(112)=2,793$, $p=0,006]$ e memória $[t(112)=2,465, p=0,015]$. As análises das médias denotam que a diferença é favorável aos estudantes de escola privada. Não houve diferenças nas tarefas de segmentação $[t(112)=0,836, p=0,405]$ e rima $[t(112)=1,717, p=0,089]$.
A análise das habilidades de crianças de 10 ano revelou não existir diferenças estatisticamente significativas nas tarefas de aliteração, segmentação e rima. A diferença foi verificada apenas na habilidade na tarefa de memória $[t(66)=-2,584, p=0,01]$; a diferença foi favorável às crianças de escolas privadas. $\mathrm{O}$ mesmo ocorreu em relação à habilidade das crianças do $3^{\circ}$ ano, ou seja, apenas foi estatisticamente significativa a diferença observada na tarefa de memória $[t(69)=-2,077, p=0,01]$. No $2^{\circ}$ ano, não ocorreram diferenças significativas em nenhuma das tarefas.

A média de habilidade encontrada nas tarefas de consciência fonológica foi de 0,45 $(D P=1,16)$, considerando que as habilidades variaram entre $-3,00$ e 2,83. Uma regressão linear (método enter) foi conduzida para determinar o efeito da escolaridade na habilidade na execução das tarefas de consciência fonológica. Foi possível perceber o efeito significativo da escolaridade $[F(1,402)=81,536, \quad p<0,0001] \quad$ e $\quad$ da idade $[F(1,402)=74,852, p<0,0001]$ na habilidade na tarefa. O modelo de regressão indica que $17 \%$ da variância na habilidade em tarefas de consciência fonológica pode ser explicada pela escolaridade, $R^{2}$ ajustado $=0,17$ e $16 \%$ pode ser explicada pela idade, $R^{2}$ ajustado $=0,16$. O beta padronizado sugere que a escolaridade ( $\beta=0,455$; SE $\beta=0,050$; padronizada $\beta=0,411)$ contribuiu um pouco mais para prever a habilidade na tarefa do que a idade $(\beta=0,350 ; \mathrm{SE}$ $\beta=0,040$; padronizada $\beta=0,396)$. Os resultados apresentaram multicolinearidade modesta entre a idade e o ano escolar, considerando-as como variáveis preditivas.

Os valores normativos para ano escolar são apresentados na Tabela 3 e 4, e as normas de habilidade nas tarefas do THPL foram elaboradas considerando a variável ano escolar e idade. Não foram elaboradas as normas por tipo de escola por ter sido observado que não houve diferenças significativas, na maioria das tarefas, por ano escolar.

Tabela 3

Percentis de Habilidades para as Tarefas de Rima, Aliteração, Segmentação e Memória Visual por Ano Escolar

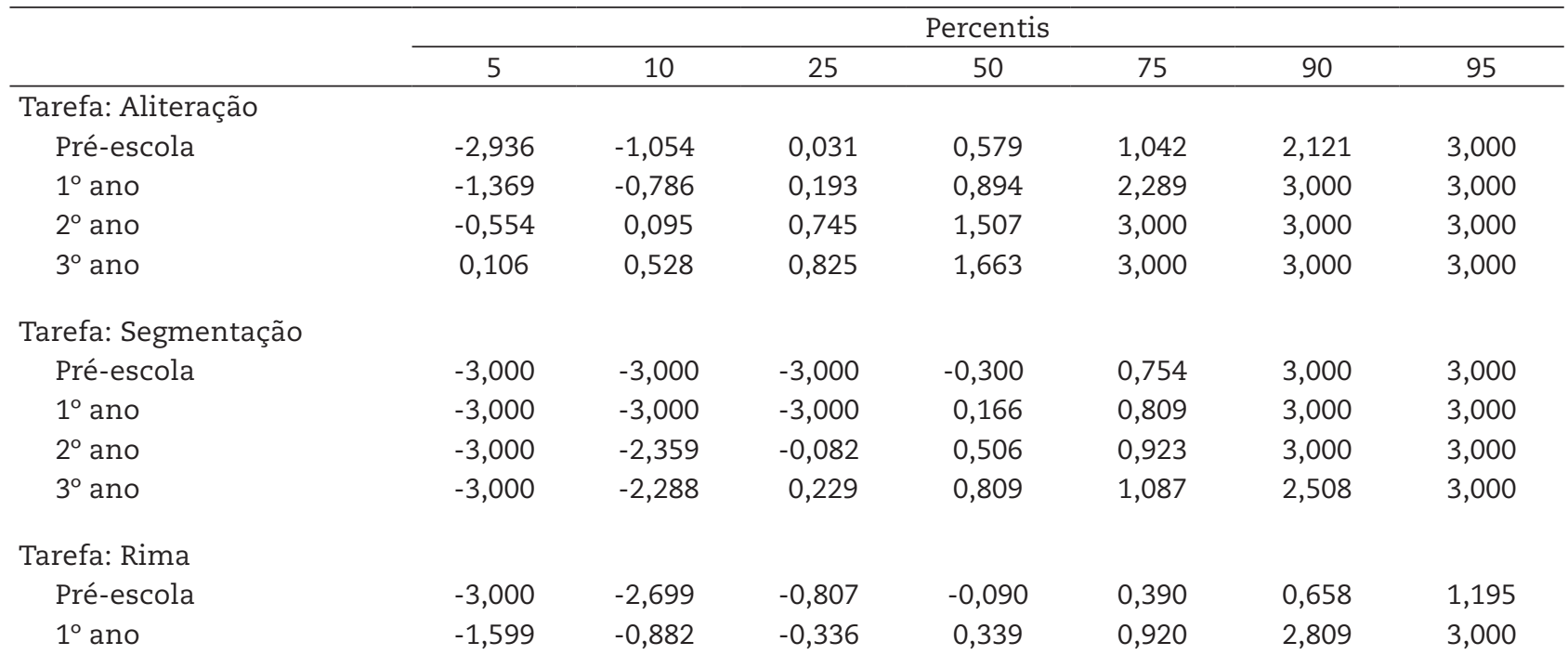


Tabela 3 (continuação)

Percentis de Habilidades para as Tarefas de Rima, Aliteração, Segmentação e Memória Visual por Ano Escolar

\begin{tabular}{|c|c|c|c|c|c|c|c|}
\hline & & & & Percent & & & \\
\hline & 5 & 10 & 25 & 50 & 75 & 90 & 95 \\
\hline $2^{\circ}$ ano & $-1,324$ & $-0,309$ & 0,311 & 0,736 & 1,762 & 3,000 & 3,000 \\
\hline $3^{\circ}$ ano & $-0,435$ & 0,067 & 0,350 & 0,787 & 2,127 & 3,000 & 3,000 \\
\hline Consciência f & & & & & & & \\
\hline Pré-escola & $-1,835$ & $-1,536$ & $-1,001$ & $-0,033$ & 0,662 & 1,129 & 1,542 \\
\hline $1^{\circ}$ ano & $-1,804$ & $-1,204$ & $-0,576$ & 0,429 & 1,220 & 1,911 & 2,204 \\
\hline $2^{\circ}$ ano & $-1,018$ & $-0,353$ & 0,389 & 0,975 & 1,622 & 2,234 & 2,302 \\
\hline $3^{\circ}$ ano & $-0,710$ & $-0,528$ & 0,633 & 1,213 & 1,879 & 2,138 & 2,375 \\
\hline Tarefa: Memó & & & & & & & \\
\hline Pré-escola & $-2,925$ & $-2,222$ & $-0,238$ & 0,866 & 2,463 & 3,000 & 3,000 \\
\hline $1^{\circ}$ ano & $-1,984$ & $-0,601$ & 0,266 & 0,933 & 3,000 & 3,000 & 3,000 \\
\hline $2^{\circ}$ ano & $-0,016$ & 0,576 & 0,867 & 3,000 & 3,000 & 3,000 & 3,000 \\
\hline $3^{\circ}$ ano & 0,695 & 0,865 & 0,874 & 3,000 & 3,000 & 3,000 & 3,000 \\
\hline
\end{tabular}

Tabela 4

Percentis de Habilidades para as Tarefas de Rima, Aliteração, Segmentação e Memória Visual por Idade

\begin{tabular}{|c|c|c|c|c|c|c|c|}
\hline & \multicolumn{7}{|c|}{ Percentis } \\
\hline & 5 & 10 & 25 & 50 & 75 & 90 & 95 \\
\hline \multicolumn{8}{|c|}{ Tarefa: Aliteração } \\
\hline 4 anos & $-3,000$ & $-1,325$ & 0,064 & 0,627 & 1,337 & 2,185 & 3,000 \\
\hline 5 anos & $-2,397$ & $-1,156$ & $-0,095$ & 0,545 & 1,064 & 3,000 & 3,000 \\
\hline 6 anos & $-1,087$ & $-0,574$ & 0,413 & 1,175 & 2,333 & 3,000 & 3,000 \\
\hline 7 anos & $-0,612$ & $-0,004$ & 0,596 & 1,380 & 3,000 & 3,000 & 3,000 \\
\hline 8 anos & $-0,062$ & 0,486 & 0,805 & 1,654 & 3,000 & 3,000 & 3,000 \\
\hline \multicolumn{8}{|c|}{ Tarefa: Segmentação } \\
\hline 4 anos & $-3,000$ & $-3,000$ & $-3,000$ & $-0,023$ & 0,841 & 3,000 & 3,000 \\
\hline 5 anos & $-3,000$ & $-3,000$ & $-3,000$ & $-0,516$ & 0,415 & 3,000 & 3,000 \\
\hline 6 anos & $-3,000$ & $-3,000$ & $-1,167$ & 0,318 & 0,849 & 3,000 & 3,000 \\
\hline 7 anos & $-3,000$ & $-3,000$ & 0,001 & 0,634 & 0,923 & 2,148 & 3,000 \\
\hline 8 anos & $-3,000$ & $-2,288$ & 0,071 & 0,726 & 1,089 & 2,656 & 3,000 \\
\hline \multicolumn{8}{|c|}{ Tarefa: Rima } \\
\hline 4 anos & $-3,000$ & $-3,000$ & $-0,874$ & $-0,216$ & 0,507 & 1,229 & 1,856 \\
\hline 5 anos & $-2,427$ & $-1,488$ & $-0,687$ & 0,102 & 0,461 & 0,989 & 2,554 \\
\hline 6 anos & $-0,887$ & $-0,656$ & $-0,078$ & 0,376 & 1,029 & 2,832 & 3,000 \\
\hline 7 anos & $-1,540$ & $-0,837$ & 0,249 & 0,669 & 1,762 & 3,000 & 3,000 \\
\hline 8 anos & $-0,445$ & $-0,048$ & 0,332 & 0,603 & 1,846 & 3,000 & 3,000 \\
\hline \multicolumn{8}{|c|}{ Consciência fonológica } \\
\hline 4 anos & $-2,038$ & $-1,714$ & $-1,013$ & 0,020 & 0,711 & 1,455 & 1,642 \\
\hline 5 anos & $-1,650$ & $-1,473$ & $-0,884$ & $-0,071$ & 0,680 & 1,223 & 1,777 \\
\hline 6 anos & $-1,390$ & $-0,977$ & $-0,323$ & 0,711 & 1,463 & 2,018 & 2,205 \\
\hline 7 anos & $-1,834$ & $-0,805$ & 0,191 & 0,769 & 1,576 & 2,227 & 2,296 \\
\hline 8 anos & $-0,701$ & $-0,436$ & 0,609 & 1,206 & 1,851 & 2,139 & 2,366 \\
\hline \multicolumn{8}{|c|}{ Tarefa: Memória } \\
\hline 4 anos & $-3,000$ & $-2,599$ & $-0,229$ & 0,867 & 1,401 & 3,000 & 3,000 \\
\hline 5 anos & $-2,189$ & $-1,130$ & $-0,060$ & 0,866 & 3,000 & 3,000 & 3,000 \\
\hline
\end{tabular}


Tabela 4 (continuação)

Percentis de Habilidades para as Tarefas de Rima, Aliteração, Segmentação e Memória Visual por Idade

\begin{tabular}{lccccccc}
\hline & \multicolumn{7}{c}{ Percentis } \\
\cline { 2 - 8 } & 5 & 10 & 25 & 50 & 75 & 90 & 95 \\
\hline anos & $-2,113$ & $-0,799$ & 0,403 & 1,033 & 3,000 & 3,000 & 3,000 \\
anos & $-1,012$ & 0,398 & 0,865 & 3,000 & 3,000 & 3,000 & 3,000 \\
anos & 0,695 & 0,865 & 1,023 & 3,000 & 3,000 & 3,000 & 3,000 \\
\hline
\end{tabular}

\section{Discussão}

O Teste de Habilidades Preditoras da Leitura (THPL) foi criado a partir da demanda de testes que avaliassem as habilidades subjacentes à leitura, com características adaptativas, computadorizado e com base na Teoria de Resposta ao Item. Além disso, é um teste de fácil manejo pelo examinando, visto que é autoaplicável, assim como é para o examinador. A vantagem que o teste oferece, além da autoaplicação, é a geração automática do desempenho do examinando em cada tarefa (habilidade, porcentagem de acerto e tempo de reação, além dos itens respondidos com os seus respectivos índices de discriminação, dificuldade e acerto casual). Com fins a facilitar o processo de interpretação do desempenho do examinando, este estudo teve por finalidade gerar dados normativos de habilidade em tarefas de aliteração, segmentação, rima e memória visual do THPL.

Estudos, como os de Carson et al. (2011) e Clemens et al. (2015), com instrumentos adaptativos computadorizados revelam diversas vantagens na sua utilização, entre as mais significativas estão a redução nos custos do material, rapidez na análise e coleta, a possibilidade de realização em grandes grupos e, principalmente, a resposta imediata dos resultados individuais e do grupo em análise. Internacionalmente, o uso de instrumentos adaptativos computadorizados está cada vez mais comum. O THPL é semelhante a instrumentos como Measures of Academic Progress (Northwest Evaluation Association, 2005, 2008, 2009), Adaptive Reading Test - STAR Early literacy (Renaissance Learning, 2015) e Istation's Indicators of Progress (ISIP) Earlu Reading (Mathes, Torgesen, \& Herron, 2016), que se baseiam na habilidade da criança e são ajustados conforme o seu nível e acurácia, tornam-se mais difíceis quando ela responde corretamente, e mais fáceis quando uma resposta incorreta é dada.

A principal utilidade clínica e educacional deste estudo é fornecer um conjunto de normas que irão auxiliar os profissionais para determinar com mais precisão o grau em que as habilidades nas tarefas de consciência fonológica (aliteração, segmentação e rima) e memória visual refletem o desempenho para o ano escolar e idade da criança. A estratificação das normas foi baseada nos achados que mostraram que a habilidade nas tarefas foi afetada pelo nível de escolaridade e na idade. As normas, portanto, foram divididas conforme o ano escolar e idade. Esses resultados são semelhantes, apesar da diferença do número amostral com os apresentados em outros estudos que utilizaram instrumentos similares, Worrell et al. (2002) em investigação com 4.112 crianças, entre pré-escola e $2^{\circ}$ ano, encontraram diferenças significativas nos anos escolares $\mathrm{e}$ idade, mas não foi verificado diferenças, por exemplo, entre os gêneros.

Ao utilizar as normas, deve-se notar que a influência do ano escolar não é equivalente nas tarefas de segmentação, rima, aliteração e memória. Em rima e aliteração, a habilidade atinge níveis altos entre a pré-escola e o $1^{\circ}$ ano e, a partir do $2^{\circ}$ ano, a habilidade estabiliza sem acréscimos significativos.

A importância da consciência fonológica (Foorman et al. (2015); Nelson, 2008) e memória (Nevo \& BarKochva, 2015; Fuchs et al. 2012) como preditores da leitura é outro valor agregado a este estudo, tendo em vista que, quanto mais cedo forem analisadas essas habilidades e mais cedo forem percebidas possíveis dificuldades, rapidamente haverá a intervenção e, provavelmente, a prevenção de dificuldades em leitura. Lipka, Katzir, e Shaul (2016) a partir de investigação longitudinal, revelam a importância de monitorar diferentes habilidades cognitivas durante os anos escolares iniciais. Os efeitos preditivos da escolaridade sobre a consciência fonológica também foram ressaltados por Gellert e Elbro (2015). O THPL, por ser adaptativo, poderia também ser utilizado para acompanhar o desenvolvimento das habilidades nas crianças avaliadas no decorrer do ano escolar.

Ademais, foram realizadas análises que verificaram a diferença entre os grupos (pré-escola, $1^{\circ}$ ano, $2^{\circ}$ ano e $3^{\circ}$ ano) para as habilidades das tarefas que compõem o THPL (rima, aliteração, segmentação e memória visual). Tais análises indicaram que há uma progressão de desempenho de habilidade para as tarefas. As crianças do $3^{\circ}$ ano apresentaram melhores habilidades nas tarefas do que as crianças da pré-escola.

Referente à habilidade dessas crianças, nota-se que não há indícios de diferenças de desempenho entre o $2^{\circ}$ ano e o $3^{\circ}$ ano escolar, atestando dessa forma a eficácia do THPL para avaliar crianças não só pré-escolares como também crianças situadas no $1^{\circ}$ ano. Esses resultados podem ser explicados por outras investigações, tais como as de Foorman et al. (2015) que, ao analisarem crianças de pré-escola até o $2^{\circ}$ ano, verificaram que, na pré-escola, 
a consciência fonológica, linguagem oral e fluência são responsáveis por $70 \%$ da variância na compreensão da leitura, no entanto, a partir do $2^{\circ}$ ano, a consciência fonológica não é mais tão preditiva.

A ausência de diferenças nas habilidades, considerando o tipo de escola (pública e privada), na maioria das tarefas analisadas por ano escolar, surpreendeu, pois acreditava-se que os alunos de escolas privadas teriam melhores resultados. Em pesquisas internacionais, não são apontadas diferenças no desempenho dessas habilidades considerando o tipo de escola. Entretanto, no Brasil, não há consenso. Por um lado, pesquisas como a de Rosal, Cordeiro, e Queiroga (2013) não verificaram diferenças significativas entre os dois grupos de crianças (escola privada versus escola particular). Por outro lado, Salles, Piccolo, Zamo, e Toazza (2013) encontraram diferenças entre os grupos em habilidades relacionadas com a leitura. Convém ressaltar que os estudos referendados não utilizaram análises de TRI e análises DIF para os itens dos testes e/ou utilizaram testes adaptativos, o que não necessariamente elimina a possibilidade de vieses na comparação. Deve-se, portanto, interpretar com cautela a ausência de diferenças entre os tipos de escola, sendo ainda necessários mais estudos sobre a temática.

A normatização preliminar do THPL será útil para a padronização do teste, que será disponibilizada no portal on-line. Os dados normativos obtidos no estudo foram integrados ao sistema do THPL e as novas aplicações do teste vão contemplar, além do desempenho da criança (página gerada automaticamente pelo sistema), a interpretação da habilidade do examinando.

\section{Referências}

Anastasi, A., \& Urbina, S. (2000). Testagem Psicológica. Porto Alegre: Artes Médicas.

Barbosa, P. M. F., Bernardes, N. G. B., Misorelli, M. I., \& Chiappetta, A. L. M. L. (2010). Relação da memória visual com o desempenho ortográfico de crianças de $2^{\mathrm{a}}$. E $3^{\mathrm{a}}$. Séries do ensino fundamental. Revista CEFAC, 12(4), 598-607.

Bridges, M. S., \& Catts, H. W. (2011). The use of a dynamic screening of phonological awareness to predict risk for reading disabilities in kindergarten children. Journal of Learning Disabilities, 44(4), 330-338. doi: 10.1177/0022219411407863

Capellini, S. A., Sampaio, M. N., Fukuda, M. T. M., Oliveira, A. M., Fadini, C. C., \& Martins, M. A. (2009). Protocolo de identificação precoce dos problemas de leitura: estudo preliminar com escolares de $1^{\circ}$ ano escolar. Revista Psicopedagogia, 26(81), $367-375$.

Capellini, S. A., \& Ciasca, S. M. (1999). Aplicação da prova de consciência fonológica (PCF) em escolares com dificuldade na leitura. Jornal Brasileiro de Fonoaudiologia, 1(1), 11-15.

Capovilla, F. C. , Macedo, E., Duduchi, M., Raphael, W. D., Guedes, M., Capovilla, A. G. S., \& Gonçalves, M. J. (1996). Avaliação computadorizada de vocabulário e compreensão auditiva em crianças falantes ou não. O Mundo da Saúde, 20(1), 421-424.

Carson, K., Gillon, G., \& Boustead, T. (2011). Computer administered versus paper-based assessment of school-entry phonological awareness ability. Asia Pacific Journal of Speech, Language, and Hearing, 14(2), 85-101. doi: 10.1179/136132811805334876

Castles, A., \& Coltheart, M.B. (2004). Is there a causal link from phonological awareness to success in learning to read? Cognition, 91(1), 77-111. doi: 10.1016/S0010-0277(03)00164-1

Clemens, N. H., Hagan-Burke, S., Luo, W., Cerda, C., Blakely, A., Frosch, J., Gamez-Patience, B., \& Jones, M. (2015). The predictive validity of a computer-adaptative assessment of kindergarten and first-grade reading skills. Scholl Psychology Review, 44(1), 76-97. doi: 10.17105/SPR44-1.76-97

Cruz, V. (2007). Uma abordagem cognitiva da leitura. Porto: Lidel Editora.

Dehaene, S. (2011). The unique role of the visual word form area in reading. Trends in Cognitive Sciences, 15(6), 254-262. doi: 10.1016/j. tics.2011.04.003

Dehaene, S. (2012). Os neurônios da leitura. Porto Alegre: Penso.

Demoulin, C., \& Kolinsky, R. (2015). Does learning to read shape verbal working memory? Psychonomic Bulletin \& Review, 1(5), 1-20. doi: 10.3758/s13423-015-0956-7

Dias, E. B. (2013). Palavras são palavras rimadas ou aliteradas: análise da relação da complexidade das propriedades da palavra no desempenho de crianças nas tarefas de detecção de rima e aliteração (Monografia). Universidade Federal da Paraíba, João Pessoa, Brasil.

Ehri, L. C. (1978). Beginning reading from a psycholinguistic perspective: Amalgamation of word identities. Em F. B. Murray (Ed.), The recognition of words (pp. 1-33), IRA Series on the Development of the Reading Process. Newark, DE: International Reading Association.

Ehri, L. C. (1979). Linguistic insight: Threshold of reading acquisition. Em T. G. Waller, \& G. E. Mackinnon (Eds.), Reading research: Advances in theory and practice (Vol. 1, pp. 66-111). New York: Academic Press.

Ehri, L. C. (1998). Research on learning to read and spell: A personal-historical perspective. Scientific Studies of Reading, 2(2), 97-114. doi: $10.1207 / \mathrm{s} 1532799 \mathrm{xssr} 0202 \_1$

Fonsaca, K. (2013). Desempenho de crianças pré-leitoras e leitoras iniciantes em tarefa de memória (Monografia). Universidade Federal da Paraíba, João Pessoa, Brasil.

Foorman, B. R., Herrera, S., Petscher, Y., Mitchell, A., \& Truckenmiller, A. (2015). The structure of oral language and reading and their relation to comprehension in Kindergarten through Grade 2. Reading and Writing: An interdisciplinary journal, 28(5), 655-681. doi: 10.1007/s11145-015-9544-5

Fuchs, D, Compton, D. L., Fuchs, L. S., Bryant, J., Hamlett, C. L., \& Lambert, W. (2012). First-grade cognitive abilities as long-term predictors of reading comprehension and disability status. Journal of Learning Disabilities, 45(3) 217-231. doi: 10.1177/0022219412442154 
Gellert, A. S., \& Elbro, C. (2015). Does a dynamic test of phonological awareness predict early reading difficulties? A longitudinal study from kindergarten through grade 1. Journal of learning disabilities, 50(3), doi: 10.1177/0022219415609185

Gerber, R. M., \& Tomitch, L. M. (2008). Leitura e cognição: Propósitos de leitura diferentes influem na geração de inferências? Acta Scientiarum. Language and Culture, 30(2), 139-147.

IBGE. (2010). Censo Demográfico. Recuperado de http://www. censo2010.ibge.gov.br

Liberman, I. Y. (1971). Basic research in speech and lateralization of language: Some implications for reading disability. Bulletin of the Orton Society, 21(1), 71-87. doi: 10.1007/BF02663712

Liberman, I. Y., Shankweiler, D., Fischer, F. W., \& Carter, B. (1974). Explicit syllable and phoneme segmentation in the young child. Journal of Experimental Child Psychology, 18, 201-212.

Liberman, I. Y., \& Shankweiler, D. (1979). Speech, the alphabet and teaching to read. Em L. Resnick \& P. Weaver (Eds.), Theory and practice of early reading (vol. 2, pp. 109-132). Hillsdale, NJ: Erlbaum.

Lipka, O., Katzir, T., \& Shaul, S. (2016). The basis of reading fluency in first grade of hebrew speaking children. Reading Fluency, 12, 91-104. doi: 10.1007/978-3-319-30478-6_6

Lundberg, I., Frost, J., \& Petersem, O. P. (1988). Effects of an extensive program for stimulating phonological awareness in preschool children. Reading Research Quarterly, 23(3), 263-268.

Mathes, P., Torgesen, J., \& Herron, J. (2016). Computer Adaptive Testing System for Continuous Progress Monitoring of Reading Growth for Students Pre-K through Grade 3. Dallas: Istation.

Minervino, C. A. M., Moita, P., Assis, G., Lucena, E., Dias, E. B., \& Fonsaca, K. (2013). Teste de habilidades preditoras da leitura (THPL). (Relatório de Pesquisa). Universidade Federal da Paraíba/Universidade de Lisboa/CNPQ, Brasil.

Minervino, C. A. M., (2000). Identificação de sinais disléxicos em pré-escolares: o pediatra pode ajudar. Pediatria Moderna, 37(1), $236-242$.

Moita, P., Minervino, C., \& Chambel, T. (2014). Tecnologia digital a favor da leitura: construção de um instrumento adaptativo informatizado. Em F. Viana, E. Ramos, E. Coquet, \& M. Martins. Li'2014, Atas do $8^{\circ}$ Encontro Internacional (10 $0^{\circ}$ Nacional) de Investigação em Leitura, Literatura Infantil \& Ilustração. (pp. 42-57). Centro de Investigação em Estudos da Criança da Universidade do Minho: Braga.

Moita, P. M. S. (2013). Avaliação Adaptativa em Dispositivos Móveis das Habilidades Cognitivas Preditoras do Desenvolvimento de Leitura em Crianças (Dissertação de mestrado). Universidade de Lisboa, Lisboa. Recuperado de http://repositorio.ul.pt/handle/10451/10320

Morais, J., Cary, L., Alegria, J., \& Bertelson, P. (1979). Does awareness of speech as a sequence of phones arise spontaneously? Cognition, 7(4), 323-331. doi: 10.1016/0010-0277(79)90020-9

Nelson, J. M. (2008). Psychometric evaluation of the mountain shadows phonemic awareness scale with a kindergarten sample. Journal of Psychoeducational Assessment, 26(3), 287-298. doi: 10.1177/0734282907306048

Nevo, E., \& Bar-Kochva, I. (2015). The relations between early working memory abilities and later developing reading skills: A longitudinal study from kindergarten to fifth grade. Mind, Brain, and Education, 9(3), 154-163. doi: 10.1111/mbe.12084

Northwest Evaluation Association. (2005). RIT scale norms for use with achievement level tests and measures of academic progress. Lake Oswego, OR: Author.

Northwest Evaluation Association. (2008). Teacher hand-book: Measures of academic progress (MAP). Lake Oswego, OR: Author. Recuperado de http://www.nwea.org/sites/www.nwea.org/files/resources/Teacher\%20Handbook_0.pdf

Northwest Evaluation Association. (2009). Technical manual for Measures of Academic Progress TM and Measures of Academic Progress for primary grades TM. Lake Oswego, OR: Author.

Partanen, M., \& Siegel, L. S. (2014). Long-term outcome of the early identification and intervention of reading disabilities. Reading and Writing, 27(4), 665-684. doi: 10.1007/s11145-013-9472-1

Pasquali, L. (2001). Técnicas de exame psicológico - TEP. Casa do Psicólogo: São Paulo.

Pereira, E. E. L. D. (2013). Análise do Desempenho de Pré-Leitores e Leitores Iniciantes em Tarefa de Segmentação (Monografia). Universidade Federal da Paraíba, João Pessoa, Brasil.

Renaissance Learning (2015). Star Assessments. EUA: Wisconsin Rapids.

Schneider, W., Küspert, P., Roth, E., Visé, M., \& Marx, H. (1997). Short- and long-term effects of training phonological awareness in kindergarten: Evidence from two German studies. Journal of Experimental Child Psychology, 66(3), 311-340. doi: 10.1006/jecp.1997.2384

Torgesen, J. K. (2002). The prevention of reading disabilities. Journal of School Psychology, 40(1), 7-26. doi: 10.1016/S0022-4405(01)00092-9

Viana, F. L. (2002). Leitura: da aprendizagem informal à aprendizagem formal. Reading: of informal learning to formal learning. (ed. 1). Porto: Edições Asa.

Worrell, F. C., Watkins, M. W., Runge, T. J., \& Hall, T. E. (2002). Pre-reading skills in Trinidad and Tobago students in the first three years of school. Caribbean Curriculum, 9, 1-19.

\section{Sobre as autoras}

Carla Alexandra da Silva Moita Minervino é estágiária pós-doutoral em Psicologia Cognitiva na Universidade Federal de Pernambuco. Professora Adjunta da Universidade Federal da Paraíba. Líder do grupo de pesquisa: Núcleo de Estudos em Saúde Mental, Educação e Psicometria (NESMEP). Vice-coordenadora da Pós-graduação em Neurociência Cognitiva e Comportamento.

Émille Burity Dias é Mestre em Neurociência Cognitiva e Comportamento. Professora substituta da Universidade Federal da Paraíba. Membro do grupo de pesquisa: Núcleo de Estudos em Saúde Mental, Educação e Psicometria (NESMEP). 